\title{
Utilidad de los índices neutrófilo/linfocito, monocito/linfocito y linfocito/plaqueta para el pronóstico de complicaciones asociadas a COVID-19
}

\author{
Christian O. Ramos-Peñafiel, ${ }^{*}{ }^{*}$ Brenda Santos-González, ${ }^{1}$ Eder N. Flores-López, ${ }^{1}$ \\ Francisco Galván-Flores, ${ }^{1}$ Lucía Hernández-Vázquez,, ${ }^{1}$ Adrián Santoyo-Sánchez, ${ }^{2}$ \\ Rosaura Montes de Oca-Yemha, ${ }^{1}$ Mónica Bejarano-Rosales, ${ }^{3}$ Érika Rosas-González, ${ }^{3}$ \\ Irma Olarte-Carrillo, ${ }^{2}$ Carlos Martínez-Murillo² y Adolfo Martínez-Tovar ${ }^{2}$

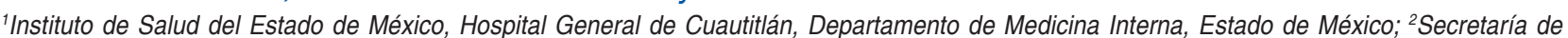 \\ Salud, Hospital General de México "Dr. Eduardo Liceaga", Departamento de Hematología, Ciudad de México; ${ }^{3}$ Secretaría de Salud, Hospital \\ General de México Dr. Eduardo Liceaga, Departamento de Nutrición Clínica, Ciudad de México. México
}

\section{Resumen}

Introducción: Diversos biomarcadores basados en conteos sanguíneos han sido de utilidad para el pronóstico de los pacientes en estado crítico por COVID-19. Objetivo: Describir la utilidad de los índices neutrófilo/linfocito (INL), monocito/linfocito (IML) y linfocito/plaqueta (IPL) para el pronóstico de la mortalidad y necesidad de soporte ventilatorio por COVID-19. Método: Cohorte retrospectiva de registros clínicos de pacientes con COVID-19 que requirieron atención hospitalaria. Resultados: Se analizaron 125 casos, la edad media fue de 51 años y 60 \%, del sexo masculino; 21.6 \% padecía diabetes mellitus tipo 2 y $18.4 \%$, hipertensión. La media de leucocitos fue $9.5 \times 10^{3} / \mathrm{LL}$ y la de neutrófilos, de $8.0 \times 10^{3} / \mu \mathrm{L}$. La media del INL fue de 12.01; del IML, de 0.442 y del IPL, de 373.07. Respecto al área bajo la curva se registraron los siguientes valores en cuanto a mortalidad: INL, 0.594; IML, 0.628 e ILP, 0.505; en cuanto a ventilación mecánica: INL, $0.581 ; I M L, 0.619$ e ILP, 0.547. En el análisis univariado, INL > $13(R M=2.750, p=0.001)$ e $I M L>0.5(R M=2.069, p=0.047)$ se asociaron a mortalidad; ILP no mostró impacto en la mortalidad ni en el soporte respiratorio. Conclusión: INL e IML son de utilidad para predecir la mortalidad en pacientes con COVID-19.

PALABRAS CLAVE: Neutrófilos. Linfocitos. Plaquetas. COVID-19. Índice pronóstico.

\section{Usefulness of the neutrophil-to-lymphocyte, monocyte-to-lymphocyte and lymphocyte- to-platelet ratios for the prognosis of COVID-19-associated complications}

\begin{abstract}
Introduction: Various biomarkers based on blood counts have been useful for the prognosis of patients critically ill with COVID-19. Objective: To describe the usefulness of the neutrophil-to-lymphocyte (NLR), monocyte-to-lymphocyte (MLR) and lymphocyte-to-platelet ([LPR) ratios for the prognosis of mortality and ventilatory support requirement for COVID-19. Method: Retrospective cohort of clinical records of patients with COVID-19 who required hospital care. Results: One-hundred and twenty-five cases were analyzed; mean age was 51 years, and $60 \%$ were of the male gender; $21.6 \%$ had type 2 diabetes mellitus, and $18.4 \%$ had hypertension. Mean leukocyte count was $9.5 \times 10^{3} / \mu \mathrm{L}$, with a neutrophil mean of $8.0 \times 10^{3} / \mathrm{ML}$. Mean NLR was 12.01, while for MLR it was 0.442 , and for $L P R, 373.07$. Regarding the area under the curve, the following
\end{abstract}

Correspondencia:

${ }^{*}$ Christian O. Ramos-Peñafiel

E-mail: leukemiachop@ hotmail.com
Fecha de recepción: 01-07-2020

Fecha de aceptación: 07-07-2020

DOI: 10.24875/GMM.20000458
Gac Med Mex. 2020;156:413-419

Disponible en PubMed

www.gacetamedicademexico.com 
values were recorded for mortality: 0.594 for NLR, 0.628 for MLR and 0.505 for $L P R$; as for mechanical ventilation, the values were 0.581 for NLR, 0.619 for MLR and 0.547 for LPR. In the univariate analysis, an NLR value > 13 (OR: 2.750, $p=0.001)$ and an MLR of $>0.5$ (OR: 2.069, $p=0.047$ ) were associated with mortality. LPR showed no impact on mortality or respiratory support. Conclusion: NLR and MLR are useful for predicting mortality in patients with COVID-19.

KEY WORDS: Neutrophils. Lymphocytes. Platelets COVID-19. Prognostic index.

\section{Introducción}

Al momento de este informe, la pandemia por coronavirus (SARS-CoV-2) se encuentra en su punto máximo de contagio (9937 618 casos en el mundo) y América Latina es la principal región afectada (2 505593 casos en Estados Unidos, 1313667 en Brasil, 275989 en Perú). ${ }^{1}$ En México, la mayor parte de los casos se concentra en la Ciudad de México y en municipios colindantes, con una mortalidad de 26381 personas. ${ }^{2}$ Acerca de los factores de riesgo, la mayor parte de la información ha sido obtenida de las diferentes series en Wuhan, China. Tanto la edad como el antecedente de hipertensión o diabetes han sido establecidos como los principales factores de riesgo clínico; ${ }^{3}$ el sexo masculino se ha asociado a mayor riesgo de complicaciones y mortalidad $(2.8 \mathrm{y}$ $1.7 \%$, respectivamente). ${ }^{4}$ Guan et al. evaluaron el impacto de la combinación de diferentes comorbilidades en 1590 pacientes de 575 hospitales en China; las neumopatías crónicas (riesgo relativo $[R R]=2.681$ ), la diabetes $(R R=1.59)$, la hipertensión $(R R=1.58)$ y el cáncer $(R R=3.5)$ fueron los principales factores de riesgo para infección por COVID-19. ${ }^{5}$ En conjunto con los datos clínicos, diversos parámetros de laboratorio se han asociado a la severidad de la enfermedad, principalmente las alteraciones en los conteos sanguíneos, la elevación de lactato deshidrogenasa y la elevación drástica de los dímeros D. ${ }^{6}$ Alteraciones como la linfopenia se han asociado a infección severa por COVID-19 (razón de momios $[\mathrm{RM}]=2.99){ }^{7}$

Existen diversos índices basados en los diferentes conteos celulares, uno de los más utilizados es el índice neutrófilo/linfocito (INL), cuya utilidad se ha extendido desde enfermedades infecciosas a inflamatorias o cáncer. ${ }^{8}$ En pacientes con neumonía comunitaria, INF $>7.2$ se han asociado a complicaciones respiratorias severas. ${ }^{9}$ En SARS-CoV-2, INL $>4$ se ha asociado a complicaciones hospitalarias e ingreso a terapia intensiva. ${ }^{10}$ Otro parámetro sanguíneo es la índice plaqueta/linfocito (IPL), cuya utilidad ha sido evaluada en enfermedades respiratorias, si bien se ha obtenido una menor área bajo la curva en comparación con INL (0.74 versus 0.88$).{ }^{11}$ El último índice utilizado es el monocito/linfocito (IML), cuya mayor utilidad se ha observado en enfermedades inflamatorias como predictor de un ataque agudo de gota u osteoartritis, en las cuales el incremento del índice se asoció a exacerbaciones articulares. ${ }^{12,13}$

La infección por COVID-19 se caracteriza por inflamación sistémica no regulada denominada "tormenta de citocinas", cuyo desarrollo se basa en la activación descontrolada de la respuesta inmune innata, lo que pone en marcha el endotelio, el complemento y el sistema de hemostasia. ${ }^{14,15}$ Esta activación modifica los diferentes conteos celulares y ocasiona linfopenia profunda, leucocitosis y neutrofilia. El objetivo de este estudio fue identificar la utilidad de los índices INL, IML, ILP en el pronóstico de pacientes con infección por COVID-19 en un hospital de recursos limitados.

\section{Método}

El Hospital General de Cuautitlán es un hospital regional que atiende a 15 municipios en el Estado de México; cuenta con 120 camas censables, de las cuales 72 fueron reconvertidas para la atención de pacientes COVID-19, se ampliaron ocho camas en al área de urgencias y la Unidad de Terapia Intensiva continuó la atención de pacientes críticos sin infección por COVID-19.

En este estudio se incluyó a pacientes hospitalizados con diagnóstico confirmado de infección por SARS-CoV-2, atendidos del 10 de abril al 15 de mayo de 2020. El tratamiento de cada caso fue acorde con el indicado por el médico tratante; la principal combinación consistió en azitromicina con hidroxicloroquina, o la adición de oseltamivir o ritonavir-lopinavir; todos recibieron tratamiento anticoagulante con heparina de bajo peso molecular. La decisión y el tiempo de inicio de soporte ventilatorio se llevó a cabo conforme a las condiciones clínicas del paciente.

El análisis de los conteos sanguíneos se realizó de manera automatizada con un citómetro modelo Beckman Coulter LH750 ${ }^{\circledR}$ (Brea, California, Estados Unidos) y el análisis de los diferentes índices se 
efectuó con los hemogramas obtenidos al ingreso hospitalario. El cálculo de los índices (INL, IML, IPL) fue ejecutado con el programa MDCalc (https://www. mdcalc.com) ${ }^{16}$

Para el análisis estadístico se utilizó el programa SPSS ${ }^{\circledR}$ versión 25 para Windows (IBM, Armonk, Nueva York, Estados Unidos), para describir la media de los valores de los conteos sanguíneos. La diferencia de los valores entre los grupos de pacientes con complicaciones se realizó mediante la prueba $t$ de Student $(p \leq 0.05$, intervalo de confianza [IC] de $95 \%$ ). Se realizó el cálculo del área bajo la curva de los tres índices respecto a las complicaciones (soporte respiratorio y muerte); al establecer el punto de corte de los índices en la mortalidad, se analizó la supervivencia mediante el método de Kaplan Meier. La diferencia entre los grupos se llevó a cabo con la prueba logrank.

\section{Resultados}

De 147 pacientes atendidos, 125 fueron incluidos en el estudio; $64 \%(n=80)$ correspondió al sexo masculino y la media de edad fue de 51.6 años, ligeramente mayor de forma no significativa en el sexo femenino (53 versus 50 años, $p=0.078$ ). Al analizar la edad por grupos, la mayoría de los pacientes se encontraba entre los 41 y 60 años $(n=72,57.6 \%)$; siguieron quienes tenían más de 60 años (23.2 \%) y los menores de 40 años (19.2\%).

Del total de casos, $18.4 \%(n=23)$ recibió atención continua en urgencias y $81.6 \%(n=102)$ ingresó al área reconvertida para COVID-19.

\section{Comorbilidades}

De los pacientes, $21.6 \%(n=27)$ presentaba diabetes mellitus tipo 2; la media del tiempo de evolución fue de 10.93 años (rango de 1 a 40), mayor en el sexo femenino (13.4 versus 8.2 años, $p=0.151$, IC $95 \%$, -2.0-12.4). El principal fármaco utilizado para el control metabólico fue la metformina $(77.8 \%, n=21)$, seguida de los diferentes tipos de insulina (11.1\%, $\mathrm{n}=3)$; dos casos recurrían a tratamiento naturista $(7.4 \%, n=2)$. El $18.4 \%(n=23)$ correspondió a pacientes con hipertensión arterial, en quienes la edad fue significativamente mayor (60 versus 49 años, $p=0.000$ ); la media de años con hipertensión fue de 12.43 (rango de 5 a 40). Los antagonistas del receptor de angiotensina constituyeron el principal tratamiento: losartán, irbersartán y telmisartán $(52.2 \%, n=12)$; siguieron los inhibidores de la enzima convertidora de angiotensina $(21.7 \%, n=5)$ : enalapril y captopril. El $26.4 \%(n=33)$ presentó ambas comorbilidades (diabetes e hipertensión) y $5.6 \%(n=7)$, solo una.

Se registraron otras comorbilidades: hipotiroidismo ( $n=2,1.6 \%)$, neumopatía crónica $(4 \%, n=5)$, epilepsia, artritis reumatoide e infección por virus de inmunodeficiencia humana (cada una en un caso, $0.8 \%)$.

\section{Hematimetría}

Las medias, rangos y diferencias del conteo hemático entre los pacientes sobrevivientes y los fallecidos se resumen en la Tabla 1; 27 casos (21.6 \%) mostraron eosinopenia absoluta al diagnóstico, en tres se identificó trombocitopenia $\left(<100 \times 10^{3} / \mu \mathrm{L}\right)$ y el conteo de plaquetas fue superior a $450 \times 10^{3} / \mu \mathrm{L}$ en seis.

\section{Eventos}

Ocurrieron 54 defunciones (43.2\%) en el seguimiento a 30 día y la media de supervivencia fue de $18.5 \pm 1.2$ días (Figura 1). El $16 \%(n=20)$ de los casos fue sometido a soporte ventilatorio.

\section{Índices sanguíneos}

Las medias de INL, IPL e IML fueron 12.01 (rango de 1.9 a 56), 373.07 (rango de 0 a 2136) y 0.442 (rango de 0 a 2.0), respectivamente. No se identificaron diferencias en las medias de los índices al estratificarlos por presencia de comorbilidades (INL: $p=0.780$, IC $95 \%$-3.109-4.1353; IPL: $p=0.415$, IC $95 \%-76.015-$ 182.88, IML: $p=0.102$, IC $95 \%-0.203-0.221$ ).

\section{Factores pronósticos}

En los pacientes que fallecieron, las medias de IML, IPL e INL fueron 0.537, 419 y 13.9, respectivamente, valores que se emplearon como puntos de corte para establecer el riesgo de ventilación mecánica, muerte y otras variables clínicas (Tabla 2). Las curvas ROC de los índices respecto a la mortalidad y uso de ventilación mecánica se muestran en las Figuras 2 y 3. INL $>13(p=0.001)$ e IML $>0.5(p=0.047)$ se relacionaron con mayor riesgo de muerte; IPL no mostró relación con la mortalidad $(p=0.432)$ ni la ventilación mecánica $(p=0.160)$. Ningún factor estudiado incrementó el riesgo de soporte ventilatorio. 
Gaceta Médica de México. 2020;156

Tabla 1. Características principales de pacientes hospitalizados con COVID-19

\begin{tabular}{|c|c|c|c|c|c|c|c|}
\hline Variable & \multicolumn{2}{|c|}{ Global $(n=125)$} & \multicolumn{2}{|c|}{ Vivo $(n=71)$} & \multicolumn{2}{|c|}{ Muerto $(n=54)$} & p \\
\hline \multirow[t]{2}{*}{ Edad en años (rango) } & \multicolumn{2}{|c|}{$51(24-85)$} & \multicolumn{2}{|c|}{$50(24-85)$} & \multicolumn{2}{|c|}{$52(25-78)$} & 0.485 \\
\hline & $\mathrm{n}$ & $\%$ & $n$ & $\%$ & $\mathrm{n}$ & $\%$ & \\
\hline $\begin{array}{l}\text { Sexo } \\
\text { Masculino } \\
\text { Femenino }\end{array}$ & $\begin{array}{l}80 \\
45\end{array}$ & $\begin{array}{l}64 \\
36\end{array}$ & $\begin{array}{l}48 \\
23\end{array}$ & $\begin{array}{l}67.6 \\
32.4\end{array}$ & $\begin{array}{l}32 \\
22\end{array}$ & $\begin{array}{l}59.3 \\
40.7\end{array}$ & 0.219 \\
\hline Diabetes & 27 & 21.6 & 20 & 28.2 & 07 & 13 & 0.032 \\
\hline \multirow[t]{2}{*}{ Hipertensión } & 24 & 19.2 & 15 & 21.1 & 09 & 16.7 & 0.348 \\
\hline & Media & Rango & Media & Rango & Media & Rango & \\
\hline Leucocitos ( $\left.\times 10^{3} / \mu \mathrm{L}\right)$ & 9.5 & $3.4-22.7$ & 9.1 & $3.4-22.7$ & 10 & $5.2-22.7$ & 0.169 \\
\hline Neutrófilos $\left(\times 10^{3} / \mu \mathrm{L}\right)$ & 8.0 & $2.1-18.5$ & 7.58 & $2.1-18.5$ & 8.5 & $3.6-18.1$ & 0.125 \\
\hline Linfocitos $\left(\times 10^{3} / \mu \mathrm{L}\right)$ & 0.91 & $0-5.76$ & 0.92 & $0.1-3.7$ & 0.90 & $0-5.76$ & 0.899 \\
\hline Monocitos $\left(\times 10^{3} / \mu \mathrm{L}\right)$ & 0.33 & $0-1.14$ & 0.30 & $0-1.14$ & 0.36 & $06-1.14$ & 0.116 \\
\hline Eosinófilos $\left(\times 10^{3} / \mu \mathrm{L}\right)$ & 0.02 & $0-0.3$ & 0.02 & $0-0.2$ & 0.02 & $0-0.3$ & 0.801 \\
\hline Hemoglobina (g/dL) & 14.9) & $7-20.8$ & 15.3 & $12.3-19.8$ & 14.3 & $7-20.8$ & 0.011 \\
\hline Plaquetas $\left(\times 10^{3} / \mu \mathrm{L}\right)$ & 233 & $71-601$ & 240.2 & $108-562$ & 223 & $71-672$ & 0.405 \\
\hline INL & 12.01 & $1.9-56$ & 10.56 & $2.0-46$ & 13.92 & $1.9-56$ & 0.052 \\
\hline IML & 0.44 & $0-2$ & 0.37 & $0-1$ & 0.53 & $0-2$ & 0.004 \\
\hline IPL & 373 & $0-2136$ & 338 & $22.5-1694$ & 419 & $0-2136$ & 0.192 \\
\hline
\end{tabular}

INL = índice neutrófilo/linfocito, IML = índice monocito/linfocito, IPL = índice plaqueta/linfocito.

Tabla 2. Asociación de las diferentes variables con el pronóstico de pacientes hospitalizados con COVID-19

\begin{tabular}{|c|c|c|c|c|c|c|}
\hline \multirow[t]{2}{*}{ Condición } & \multicolumn{3}{|c|}{ Muerte } & \multicolumn{3}{|c|}{ Soporte ventilatorio } \\
\hline & RM & IC $95 \%$ & $p$ & RM & IC $95 \%$ & $p$ \\
\hline Diabetes & 0.379 & $0.1472-0.9797$ & 0.045 & 0.891 & $0.2714-2.9277$ & 0.849 \\
\hline Comorbilidades & 0.486 & $0.2222-1.0672$ & 0.072 & 1.124 & $0.4113-3.0740$ & 0.819 \\
\hline Sexo masculino & 0.697 & $0.3339-1.4550$ & 0.336 & 1.053 & $0.3870-2.8671$ & 0.919 \\
\hline Edad > 50 años & 1.790 & $0.8657-3.7049$ & 0.116 & 3.636 & $1.1392-11.6077$ & 0.029 \\
\hline Linfocitos $<0.5$ & 0.638 & $0.2772-1.4711$ & 0.292 & 3.477 & $1.2707-9.5153$ & 0.015 \\
\hline Leucocitos > 10 & 1.565 & $0.6798-3.6074$ & 0.292 & 1.528 & $0.5281-4.4205$ & 0.434 \\
\hline INL $>13$ & 2.750 & $1.2690-5.9593$ & 0.001 & 1.953 & $0.7362-5.1814$ & 0.178 \\
\hline $\mathrm{IML}>0.5$ & 2.069 & $1.0082-4.2497$ & 0.047 & 1.851 & $0.6990-4.9016$ & 0.215 \\
\hline $\mathrm{IPL}>419$ & 1.472 & $0.676-3.207$ & 0.330 & 1.411 & $0.512-3.888$ & 0.505 \\
\hline
\end{tabular}

$\mathrm{RM}=$ razón de momios, IC = intervalo de confianza, INL = índice neutrófilo/linfocito, IML = índice monocito/linfocito, IPL = índice plaqueta/linfocito.

El análisis diferenciado de las curvas se supervivencia indica que INL $>13$ (logrank = 0.004) e IML > 0.5 (logrank $=0.055)$ se asociaron significativamente a menor supervivencia y que ILP no impactó significativamente en la supervivencia (logrank $=0.374$ ), como se observa en la Figura 1 ( $B, C$ y $D$, respectivamente). 

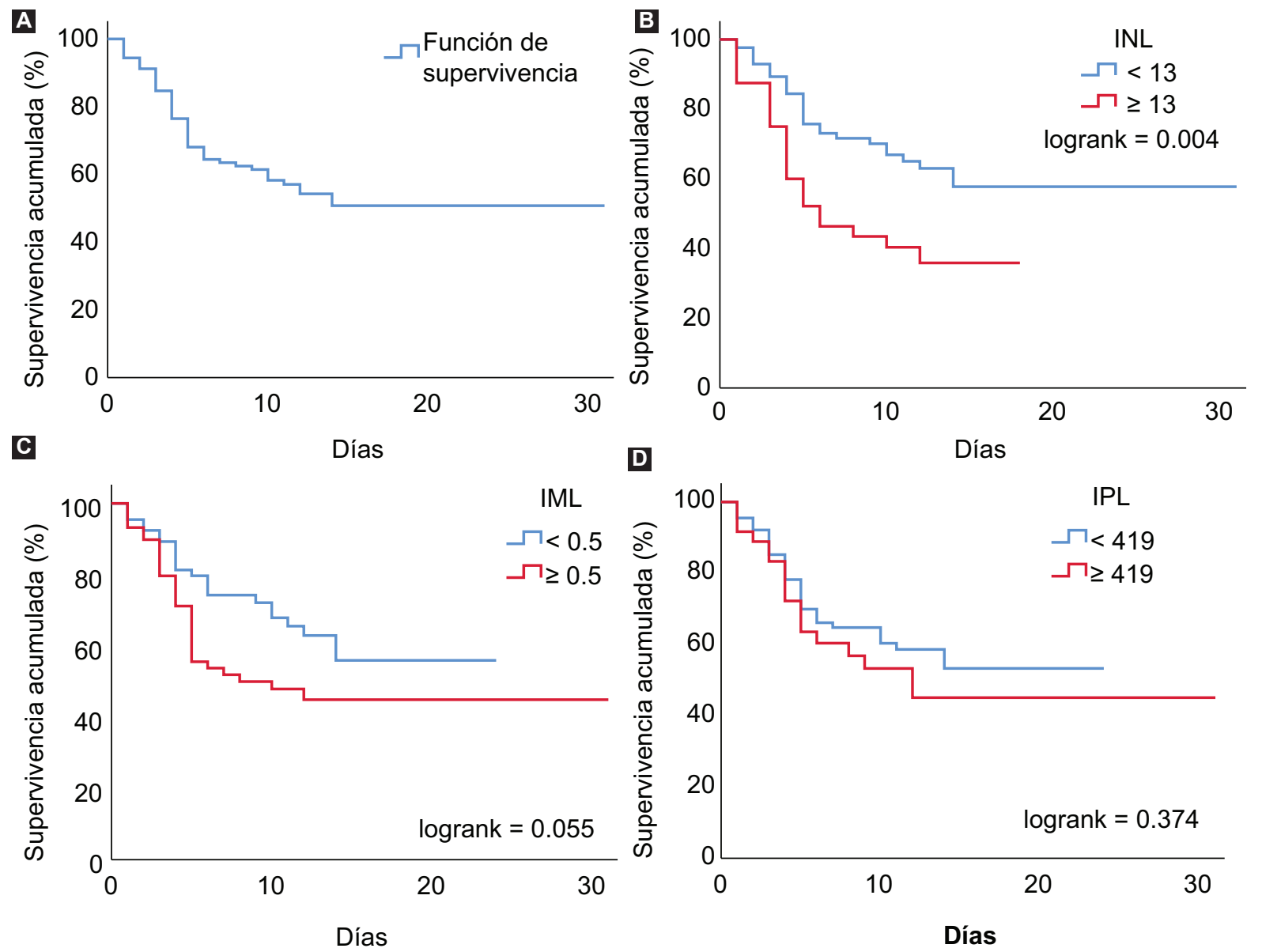

Figura 1. Supervivencia de pacientes hospitalizados por COVID-19. A) Curva de supervivencia. B) Curva de supervivencia COVID-19 estratificada por INL; C) curva de supervivencia COVID-19 estratificada por IML; D) curva de supervivencia COVID-19 estratificada por IPL. INL = índice neutrófilo/linfocito, IML = Índice monocito/linfocito, IPL = índice plaqueta/linfocito.

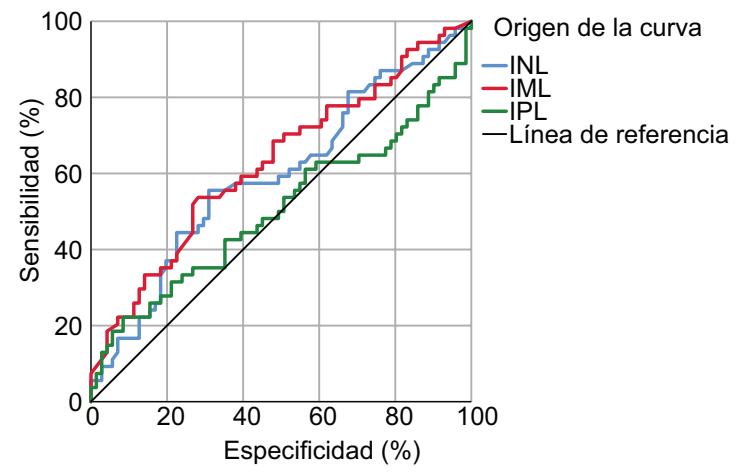

Figura 2. Curva ROC de los tres índices respecto al pronóstico de mortalidad en pacientes hospitalizados por COVID-19.

\section{Discusión}

A pesar de las medidas implementadas en el sistema de salud, gran parte de los centros hospitalarios cuenta solo con las pruebas de laboratorio de

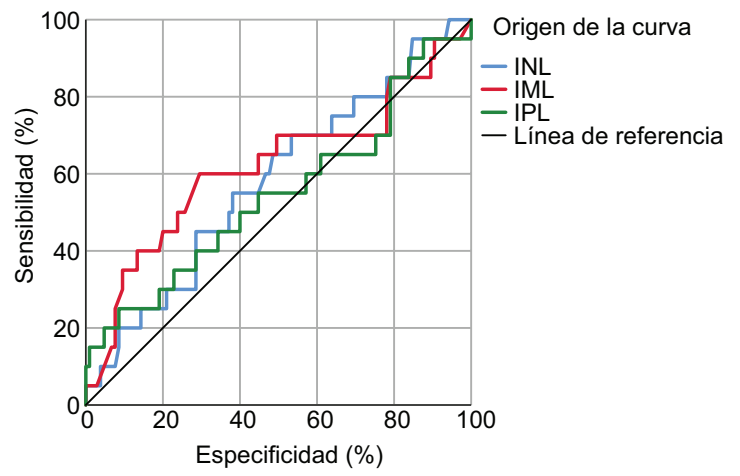

Figura 3. Curva ROC de los tres índices respecto al pronóstico de soporte ventilatorio en pacientes hospitalizados por COVID-19.

rutina básicas para la atención de los pacientes con infección por COVID-19. Entre estas pruebas, la hematimetría ha sido de gran utilidad para identificar a los pacientes con riesgo potencial de complicaciones. El objetivo principal del presente estudio fue 
evaluar la utilidad de tres índices sanguíneos de fácil obtención y bajo costo, en el pronóstico de las complicaciones asociadas a SARS-CoV-2. Tanto INL como IML fueron de utilidad para identificar a los pacientes con riesgo mayor de muerte, a diferencia ILP, el cual contó con menor área bajo la curva y no sirvió para predecir los casos que requirieron soporte ventilatorio. La presencia de linfopenia severa se asoció directamente a soporte ventilatorio.

Los hallazgos anteriores concuerdan con los de Zhang et al., quienes reportaron que la linfopenia fue un dato constante en 663 pacientes con COVID-19, en especial en los pacientes críticos (87.2\%) que requirieron soporte ventilatorio. ${ }^{17}$ Por su parte, Wang et al. evaluaron el impacto de diferentes variables clínicas y de laboratorio en el pronóstico; en su serie, la cuenta de leucocitos fue ligeramente mayor en los pacientes que fallecieron (4.4 versus $6.7 \times 10^{3} / \mu \mathrm{L}$, $p=0.004)$, al igual que la de neutrófilos (2.8 versus $5.4 \times 10^{3} / \mu \mathrm{L}, \mathrm{p}=0.001$ ); en el modelo univariado, tanto la edad como el sexo constituyeron factores independientes de riesgo. ${ }^{18}$ En nuestros resultados, tanto la edad como el sexo masculino se comportaron como factores de riesgo para mortalidad.

En cuanto a los índices, INL fue uno de los principales para predecir complicaciones asociadas a COVID-19. Este índice refleja el estado inflamatorio del individuo y es de gran utilidad en neumonías bacterianas cuyo valor concuerda con puntuaciones como las de CURB65..$^{19}$ En neumonía bacteriana, INL oscila entre 8 y 13, a diferencia de la infección por tuberculosis, cuyo valor es menor a 7.20-22 En la infección por SARS-CoV-2, el índice se encuentra entre los biomarcadores propuestos de severidad en conjunto con la proteína $C$ reactiva, péptido amiloide sérico, interleucina-6, lactato deshidrogenasa, dímero $D$, troponina y algunos biomarcadores renales. ${ }^{23}$ Liu et al. identificaron que INL se asoció independientemente a mortalidad: los casos con un valor de entre 4.85 a 88.09 se asociaron significativamente a complicaciones respiratorias, con $\mathrm{RM}=16.04(p=0.0001) .{ }^{24}$ Recientemente en Louisiana (Estados Unidos), Tatum et al. registraron que este índice (con punto de corte de 4.94) se relacionó con el riesgo de uso de soporte ventilatorio y de mortalidad hospitalaria. ${ }^{25}$

Finalmente, respecto a IML e IPL no se ha establecido su utilidad en pacientes con COVID-19. En nuestro estudio, el índice que demostró mayor utilidad fue IML, debido principalmente al papel de los monocitos sobre el estado de inflamación inicial asociado a COVID-19. Lu et al. describieron el comportamiento evolutivo del hemograma en COVID-19, en donde los conteos de monocitos, eosinófilos y linfocitos mostraron una recuperación después del día 15 de iniciado el padecimiento. ${ }^{26}$ Los datos apoyan el papel de la monitorización de estos índices no solo al diagnóstico, sino durante el seguimiento de la enfermedad.

Actualmente, la infección por SARS-CoV-2 se encuentra en su pico máximo en América Latina, a pesar de las estrategias implementadas por los diferentes ministerios de salud; aún existen hospitales que cuentan solo con estudios de rutina para el monitoreo de la enfermedad. Los índices basados en los conteos sanguíneos son de fácil acceso; INL e IML pueden predecir la mortalidad asociada a infección por COVID-19.

\section{Agradecimientos}

Un afectuoso y respetuoso agradecimiento a todo el personal de las unidades médicas (enfermeras, médicos, encargados del control de infecciones, laboratoristas, recepcionistas y demás colaboradores) que continúa ofreciendo la mejor atención posible, haciendo hasta lo imposible con los escasos recursos con que dispone en ocasiones. Un merecido reconocimiento póstumo al personal de enfermería del Hospital de Cuatitlán que lamentablemente ha perdido la vida en esta pandemia.

\section{Conflicto de intereses}

Los autores declaran no tener conflicto de intereses alguno.

\section{Financiamiento}

Los autores no recibieron patrocinio para llevar a cabo este artículo.

\section{Responsabilidades éticas}

Protección de personas y animales. Los autores declaran que los procedimientos se llevaron a cabo de conformidad con las normas éticas del comité de experimentación humana responsable y de acuerdo con la Asociación Médica Mundial y la Declaración de Helsinki.

Confidencialidad de los datos. Los autores declaran que han seguido los protocolos de su centro de trabajo sobre la publicación de datos de pacientes. 
Derecho a la privacidad y consentimiento informado. Los autores declaran que en este artículo no aparecen datos de pacientes.

\section{Bibliografía}

1. Johns Hopkins University School of Medicine [Internet]. EE. UU.: COVID-19 map; 2020.

2. Secretaria de Salud [Internet]. México: Coronavirus (COVID-19)-Comunicado técnico diario; 2020.

3. Zhou F, Yu T, Du R, Fan G, Liu Y, Liu Z, et al. Clinical course and risk factors for mortality of adult inpatients with COVID-19 in Wuhan, China: a retrospective cohort study. Lancet. 2020;395:1054-1062.

4. Gebhard C, Regitz-Zagrosek V, Neuhauser HK, Morgan R, Klein SL. Impact of sex and gender on COVID-19 outcomes in Europe. Biol Sex Differ. 2020;11:9.

5. Guan WJ, Liang WH, Zhao Y, Liang HR, Chen ZS, Li YM, et al. Comorbidity and its impact on 1,590 patients with Covid-19 in China: a nationwide analysis. Eur Respir J. 2020;55:2000547.

6. Li X, Xu S, Yu M, Wang K, Tao Y, Zhou Y, et al. Risk factors for severity and mortality in adult COVID-19 inpatients in Wuhan. J Allergy Clin Immunol. 2020;146:110-118.

7. Zhao Q, Meng M, Kumar R, Wu Y, Huang J, Deng Y, et al. Lymphopenia is associated with severe coronavirus disease 2019 (COVID-19) infections: a systemic review and meta-analysis. Int $\mathrm{J}$ Infect Dis. 2020:96:131-135.

8. Gürağaç A, Demirer Z. The neutrophil-to-lymphocyte ratio in clinical practice. Can Urol Assoc J. 2016;10:141.

9. Che-Morales J, Cortés-Télles A. Neutrophil-to-lymphocyte ratio as a serum biomarker associated with community acquired pneumonia. Rev Med Inst Mex Seguro Soc. 2018;56:537-543.

10. Ciccullo A, Borghetti A, Dal Verme LZ, Tosoni A, Lombardi F, Garcovich M, et al. Neutrophil-to-lymphocyte ratio and clinical outcome in COVID-19: a report from the Italian front line. Int J Antimicrob Agents. 2020;56:106017.

11. Kurtipek E, Tahir-Bekci T, Kesli R, Sami-Sami S, Terzi Y. The role of neutrophil-lymphocyte ratio and platelet-lymphocyte ratio in exacerbation of chronic obstructive pulmonary disease. J Park Med Assoc. 2015;65:1283-1287.
12. Kadiyoran C, Zengin O, Cizmecioglu HA, Tufan A, Kucuksahin O, Cure $\mathrm{MC}$, et al. Monocyte to lymphocyte ratio, neutrophil to lymphocyte ratio, and red cell distribution width are the associates with gouty arthritis. Acta Medica (Hradec Kralove). 2019;62:99-104.

13. Gao K, Zhu W, Liu W, Ma D, Li H, Yu W, et al. Diagnostic value of the blood monocyte-lymphocyte ratio in knee osteoarthritis. J Int Med Res. 2019;47:4413-4421.

14. Coperchini F, Chiovato L, Croce L, Magri F, Rotondi M. The cytokine storm in COVID-19: an overview of the involvement of the chemokine/ chemokine-receptor system. Cytokine Growth Factor Rev. 2020;53:25-32.

15. Colling ME, Kanthi Y. COVID-19-associated coagulopathy: an exploration of mechanisms. Vasc Med. 2020.

16. MDCalc [En línea]. EEUU: MDCalc; 2020.

17. Zhang J, Wang X, Jia X, Li J, Hu K, Chen G, et al. Risk factors for disease severity, unimprovement, and mortality in COVID-19 patients in Wuhan, China. Clin Microbiol Infect. 2020;26(6):767-772.

18. Wang D, Yin Y, Hu C, Liu X, Zhang X, Zhou S, et al. Clinical course and outcome of 107 patients infected with the novel coronavirus, SARS-CoV-2, discharged from two hospitals in Wuhan, China. Crit Care. 2020:24:188.

19. de Jager CPC, Wever PC, Gemen EFA, Kusters R, van Gageldonk-Lafeber $A B$, van der Poll $T$, et al. The neutrophil-lymphocyte count ratio in patients with community-acquired pneumonia. PLoS One. 2012;7:e46561.

20. Curbelo J, Rajas O, Arnalich B, Galván-Román JM, Luquero-Bueno S, Ortega-Gómez M, et al. Neutrophil count percentage and neutrophil-lymphocyte ratio as prognostic markers in patients hospitalized for community-acquired pneumonia. Arch Bronconeumol. 2019;55:472-477.

21. Yoon NB, Son C, Um SJ. Role of the neutrophil-lymphocyte count ratio in the differential diagnosis between pulmonary tuberculosis and bacterial community-acquired pneumonia. Ann Lab Med. 2013;33:105-110.

22. Cataudella E, Giraffa CM, Di Marca S, Pulvirenti A, Alaimo S, Pisano M, et al. Neutrophil-to-lymphocyte ratio: an emerging marker predicting prognosis in elderly adults with community-acquired pneumonia. J Am Geriatr Soc. 2017:65:1796-1801.

23. Kermali M, Khalsa RK, Pillai K, Ismail Z, Harky A. The role of biomarkers in diagnosis of COVID-19-A systematic review. Life Sci. 2020;254:117788.

24. Liu Y, Du X, Chen J, Jin Y, Peng L, Wang HHX, et al. Neutrophil-to-lymphocyte ratio as an independent risk factor for mortality in hospitalized patients with COVID-19. J Infect. 2020;81:e6-e12.

25. Tatum D, Taghavi S, Houghton A, Stover J, Toraih E, Duchesne J. Neutrophil-to-lymphocyte ratio and outcomes in Louisiana Covid-19 patients. Shock. 2020.

26. Lu G, Wang J. Dynamic changes in routine blood parameters of a severe COVID-19 case. Clin Chim Acta. 2020;508:98-102. 